\title{
System for Simulating Dynamic Features of Crowd Behavior
}

\author{
Kai Yip Wong* \\ University College London
}

Mary-Ann Thyvetil

University College London

\section{Andriana Machaira}

University College London
Celine Loscos ${ }^{\dagger}$

University College London

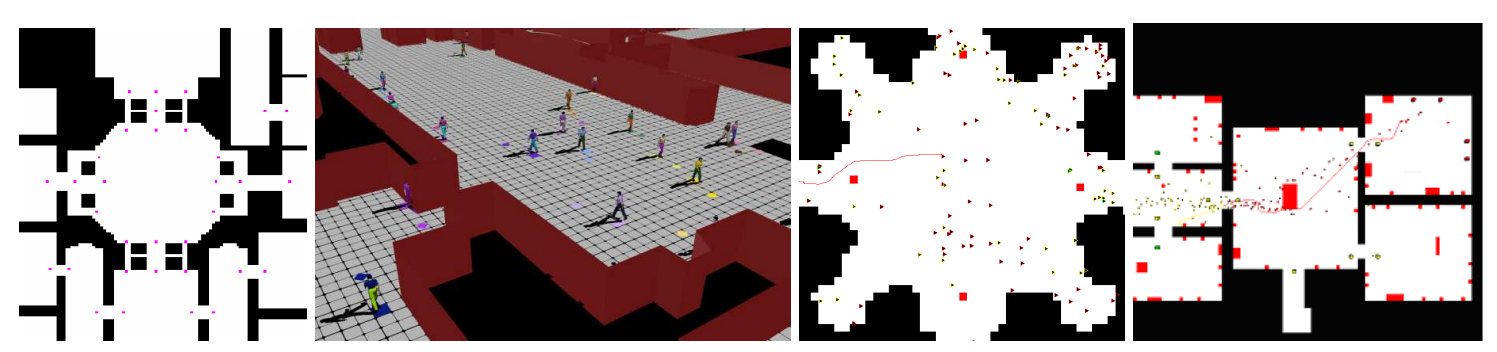

Figure 1: Left to right: Goals positioned in doorways, 3D museum representation, normal condition crowd behavior, agent evacuation.

CR Categories: I.3.7 [Computer Graphics]: Three-Dimensional Graphics and Realism-Animation-;

\section{Introduction}

The incorporation of virtual populations into virtual environments enhances the realism of these environments. Many effective algorithms have been developed to govern the movement of individual avatars. One [Loscos et al. 2003] combines local navigation rules based on a multi-layer data system as in [Tecchia et al. 2001] while using goals for way finding. We designed a new system building on those ideas that adds new levels to this behavior in the form of points of attraction, evacuations and walking in small groups.

\section{Navigation}

In our system, navigation is simulated at two different levels. Agents make local decisions by accessing information stored in 2D maps, for collision detection with the environment and other agents. Their overall direction is controlled through a graph of goals placed at junctions, where decisions are made for new directions. Between two or more rooms or streets, the agents are faced with a decision that is biased in favor of the direction with higher priority. This reflects the fact that a population is rarely evenly distributed over the entire accessible area but is influenced by the environment layout and the personal preferences of the individuals [Penn and Turner 2001]. The result is that the average density in each room converges with the higher priority rooms containing a higher density of agents. The idea is similar to [Michael and Chrisanthou 2003], while its implementation is very different.

\section{Attractors}

As mentioned previously the simulation uses 2D maps to store the height of the environment and classify which parts of the floor are accessible or otherwise. Thus the individual cells of the maps encode instructions informing any passing agents of the environment and invoking the correct behavioral reaction. Similarly, attractors

\footnotetext{
*e-mail: yip.wong@cs.ucl.ac.uk

†e-mail:c.loscos@cs.ucl.ac.uk
}

associated with callbacks can be used to simulate specific behaviors e.g. pedestrian crossings can be represented as tiles that inform passing agents of the boundary between the accessible pavement and the ground in-between. In an indoor environment the attractors represent points of interest and act like gradient potential fields with the cells around drawing agents toward its position.

\section{Evacuation}

With small adjustments to the navigation algorithm the simualation can be extended to a simple evacuation system. The graph of goals can be adjusted to add doorways and emergency exits. New priority rules are assigned to each goal depending upon how many rooms need to be traversed to reach the nearest exit. Choosing the next visible goal with a higher priority, the agents can find the logical path towards the nearest exit.

\section{Group Behavior}

In most simulation systems humans are considered either as individual or herds. In reality humans navigate either as individual or as small groups of 2 to 4 people. In our system we use a model combining both clustering and spring force that enables the agents to move in an organized formation. The clustering involves setting the same direction and speed to every group member to keep them close together. The attractive and repulsive forces make slight alterations to the agents' positions to keep them in a side-by-side formation that is more natural.

\section{Conclusion}

We presented a system that allows a variety of behavior for crowds in large scale environments. The simulation runs in real-time and permits a navigation based on complex rules while allowing collision detection, attractor features and evacuation for agents walking alone or in pairs. Each of the features can be invoked simultaneously, although one must be careful to avoid direction conflicts caused by attractors in an evacuation situation.

\section{References}

Loscos, C., Marchal, D., AND Meyer, A. 2003. Intuitive crowd behaviour in dense urban environments using local laws. TPCG, IEEE Computer Society Press.

Michael, D., And Chrisanthou, Y. 2003. Automatic high level avatar guidance based on the affordance of movement. Eurographics (September).

Penn, A., And Turner, A. 2001. Space syntax based agent models. Pedestrian and Evacuation Dynamics, 99-114.

Tecchia, F., Loscos, C., Conroy, R., and Chrysanthou, Y. 2001. Agent behaviour simulator (abs); a platform for urban behaviour development. GTEC. 